\title{
Vortex Guidance and Transport in Channeled Pinning Arrays
}

\author{
C. Reichhardt and C. J. O. Reichhardt \\ Theoretical Division and Center for Nonlinear Studies, \\ Los Alamos National Laboratory, Los Alamos, New Mexico 87545, USA
}

(Dated: December 19, 2019)

\begin{abstract}
We numerically examine vortices interacting with pinning arrays where a portion of the pinning sites have been removed in order to create coexisting regions of strong and weak pinning. The region without pinning sites acts as an easy-flow channel. For driving in different directions with respect to the channel, we observe distinct types of vortex flow. When the drive is parallel to the channel, the flow first occurs in the pin free region followed by a secondary depinning transition in the pinned region. At high vortex densities there is also an intermediate plastic flow phase due to the coupling between the weak and strong pinning regions. For driving applied perpendicular to the channel, we observe a jammed phase in which vortices accumulate on the boundary of the pinned region due to the vortex-vortex repulsion, while at higher drives the vortices begin to flow through the pinning array. For driving at an angle to the channel, depending on the filling we observe a drive-induced reentrant pinning effect as well as negative differential mobility which occurs when vortices move from the unpinned to the pinned portion of the sample.
\end{abstract}

\section{INTRODUCTION}

Depinning and sliding dynamics of an assembly of particles in the presence of either a random or a periodic landscape occurs in a wide variety of condensed matter systems, including vortices in type-II superconductors ${ }^{1,2}$, sliding Wigner crystals ${ }^{3}$, skyrmions ${ }^{4,5}$, colloids ${ }^{6.7}$, and frictional dynamics 8 . In most of these systems the disorder is generally uniform, but there are some cases in which the pinning is anisotropic or spatially inhomogeneous, where regions of strong pinning coexist with regions of weak pinning. An example of this is vortices in type-II superconducting samples that have thicknesses modulations ${ }^{9-20}$. Here the pinning is anisotropic, and the vortices can slide easily in one direction but not in the other. When a driving current is applied such that the resulting force on the vortices is aligned with the easy flow direction, the pinning is stronger in thick regions than in thin regions, and the vortices preferentially flow in the weak pinning regions past other vortices that are trapped in the strong pinning regions ${ }^{10,11,17}$. It is also possible to drive vortices at an angle with respect to a pinning array in order to produce a combination of guided flow and plastic vortex flow ${ }^{12,13,18,20}$. Large scale regions of strong pinning coexisting with weak pinning can be created using masked irradiation techniques $21-26$. In soft matter and magnetic systems, inhomogeneous pinning can appear when one part of the sample contains strong disorder and the other part of the sample contains weak disorder 27,28 . Previous work on inhomogeneous pinning has generally focused on the case when the particles are driven parallel to an easy flow direction of the pinning, which produces shear banding or gradients in the particle velocity.

Advances in nanostructuring have now made it possible to create carefully controlled pinning landscapes. For vortices in type-II superconductors, there are numerous ways to generate periodic pinning structures ${ }^{29-33}$, so it should be feasible to create samples containing strips of periodic pinning coexisting with regions in which there are no artificial pinning sites. The vortex dynamics in such a system should have several interesting features, since even in systems with uniform periodic pinning, a rich variety of dynamic phases can arise depending on the ratio of the number of vortices to the number of pinning sites, the direction of the drive with respect to the pinning array symmetry, and the size of the pining sites $34-38$.

In this work we numerically examine a superconducting system in which one half of the sample contains a square pinning array and the other half contains no pinning. The initial vortex density in the absence of driving is uniform across the system. Under an applied drive we observe very different dynamical phases depending on whether the driving direction is parallel, perpendicular, or at an angle to the pinning stripe. These dynamical phases are associated with distinct features in the velocity-force curves and vortex flow patterns. The dynamics also depends strongly on the ratio of the number of vortices to the number of pinning sites. When an increasing drive is applied parallel to the pinning strip, flow of only the vortices in the unpinned region is followed by plastic flow at the interface between the pinned and unpinned regions and then by a flow of all the vortices in the entire sample. For perpendicular driving, we observe a jammed phase in which vortices accumulate along the edge of the pinned portion of the sample. The most interesting regime is for driving at angle. Here, the vortex motion is confined to the pin-free channel and guided by the pinning strip for low drives, but at sufficiently high drives, moving vortices begin to enter the strong pinning region, producing either a reentrant pinned phase or negative differential mobility. We map out these phases in a series of dynamic phase diagrams. Our results could be tested with nanostructured superconductors, and should also be general to other assemblies of interacting particles in inhomogeneous pinning. 

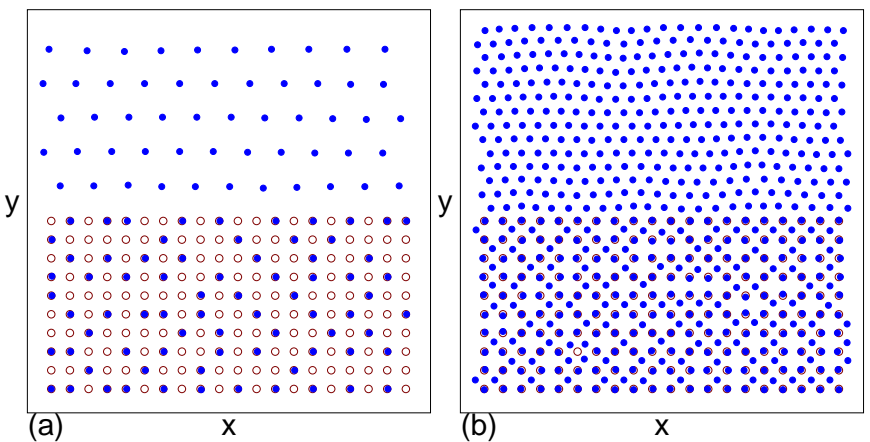

FIG. 1. The vortex positions (filled circles) and pinning site positions (open circles) in a system where half of the sample contains a square pinning array and the other half of the sample contains no pinning. (a) $B / B_{\phi}=1 / 3$, where there are fewer vortices than pinning sites. (b) $B / B_{\phi}=1.67$, where there are more vortices than pinning sites and interstitial vortices appear within the pinned region.

\section{SIMULATION}

We model the vortices as individual point particles in a two dimensional system of size $L \times L$ with periodic boundary conditions. The lower half of the sample contains a square pinning array with lattice constant $a$, while the upper half of the sample is pin-free. The interface between the pinned and unpinned regions is along the $x$ direction. There are $N_{p}$ pinning sites and $N_{v}$ vortices, and the vortex density is given by $B=N_{v} / L^{2}$. We define the matching vortex density $B_{\phi}$ to be the density at which the number of vortices would be equal to the number of pinning sites if the entire system were filled with pinning. Thus, in our case, at $B=B_{\phi}, N_{v}=2 N_{p}$. The initial vortex positions are obtained by annealing from a high temperature down to $T=0$, which creates a uniform vortex density with a roughly equal number of vortices in the pinned and pin-free regions of the sample. In Fig. 1 we show a snapshot of the vortex configurations after annealing for two different vortex densities. At $B / B_{\phi}=1 / 3$ in Fig. 1(a), a portion of the pinning sites are empty, while at $B / B_{\phi}=1.67$ in Fig. 1(b), all of the pinning sites are occupied and a portion of the vortices in the pinned region are located at interstitial positions between the pinning sites. In both cases, the vortices in the pin-free region form a hexagonal lattice.

The vortex dynamics for vortex $i$ is obtained using the following equation of motion:

$$
\alpha_{d} \mathbf{v}_{i}=\mathbf{F}_{i}^{s s}+\mathbf{F}_{i}^{p}+\mathbf{F}_{i}^{D} .
$$

Here the repulsive vortex-vortex interaction force is $\mathbf{F}_{i}=$ $\sum_{j=1}^{N} K_{1}\left(r_{i j}\right) \hat{\mathbf{r}}_{i j}$, where $\mathbf{v}$ is the vortex velocity, $\alpha_{d}=1.0$ is the damping term, $r_{i j}=\left|\mathbf{r}_{i}-\mathbf{r}_{j}\right|$ and $K_{1}(r)$ is the modified Bessel function which falls off exponentially for large $r$. A uniform driving force $\mathbf{F}^{D}=F_{D} \hat{\alpha}$ is applied to all the vortices, with $\hat{\alpha}=\hat{\mathbf{x}}$ for driving parallel to the pinning stripe, $\hat{\alpha}=\hat{\mathbf{y}}$ for driving perpendicular to the pinning

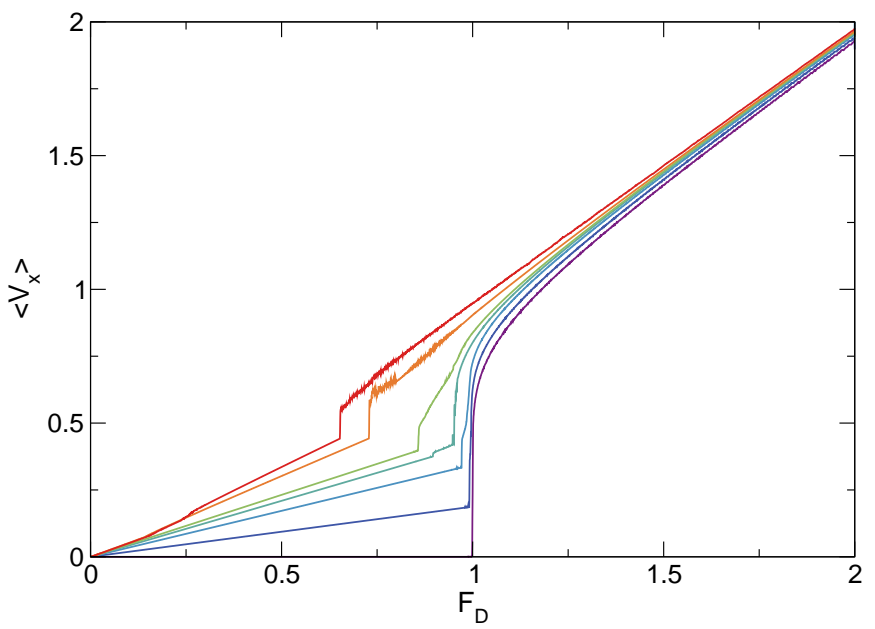

FIG. 2. $\left\langle V_{x}\right\rangle$ vs $F_{D}$ for the system in Fig. 1 with parallel driving at $B / B_{\phi}=0.083,0.167,0.33,0.5,0.67,1.33$, and 1.67 , from bottom to top.
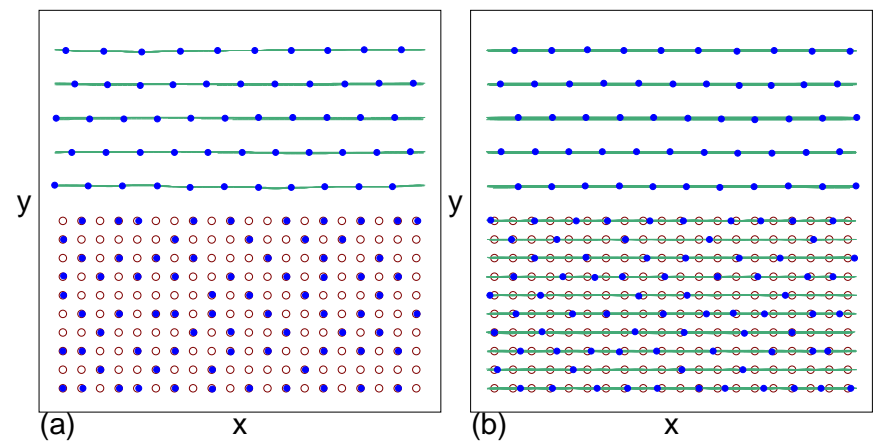

FIG. 3. The vortex positions (filled circles), pinning site positions (open circles), and vortex trajectories (lines) for the system in Fig. 2 with parallel driving at $B / B_{\phi}=0.33$. (a) At $F_{D}=0.5$, only the vortices in the unpinned region flow. (b) At $F_{D}=1.1$, all the vortices are flowing.

stripe, and $\hat{\alpha}=\cos (\theta) \hat{\mathbf{x}}+\sin (\theta) \hat{\mathbf{y}}$ for driving at an angle $\theta$ to the pinning stripe. The pinning sites are modeled as finite range attractive parabolic potential wells with a radius of $r_{p}$ which exert a maximum pinning force of $F_{p}$ on a vortex. In the single vortex limit, depinning occurs when $F_{D} / F_{p}>1.0$. In this work we fix $B_{\phi}=0.3$, $F_{p}=1.0$, and $r_{p}=0.35$, and we vary the number of vortices or filling factor $N_{v} / N_{p}$ as well as the direction of the applied drive. For each value of $F_{D}$, we measure the average velocity in the $x$ direction, $\left\langle V_{x}\right\rangle=N_{v}^{-1} \sum_{i}^{N_{v}} \mathbf{v}_{i} \cdot \hat{\mathbf{x}}$, or the $y$ direction, $\left\langle V_{y}\right\rangle=N_{v}^{-1} \sum_{i}^{N_{v}} \mathbf{v}_{i} \cdot \hat{\mathbf{y}}$.

\section{DRIVING IN THE PARALLEL DIRECTION}

We first consider driving in the easy flow or $x$-direction. In Fig. 2 we plot $\left\langle V_{x}\right\rangle$ versus $F_{D}$ for samples with $B / B_{\phi}=0.08,0.167,0.33,0.5,0.67,1.33$, and 1.67 . 

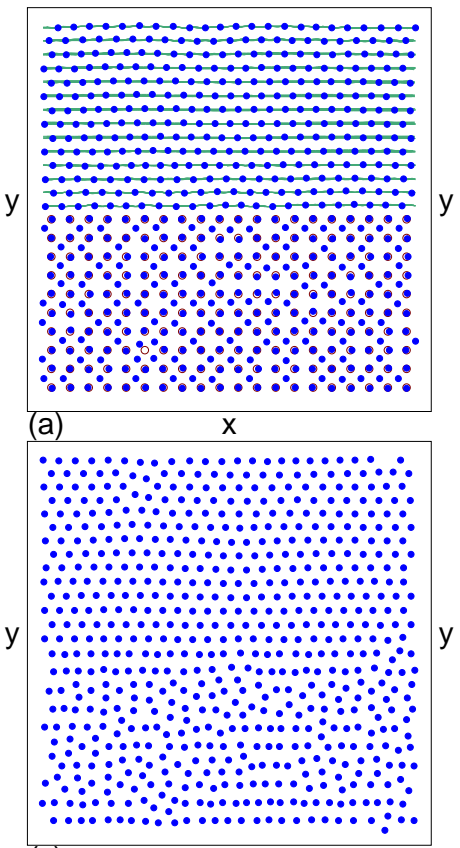
(c)

$$
\mathrm{X}
$$

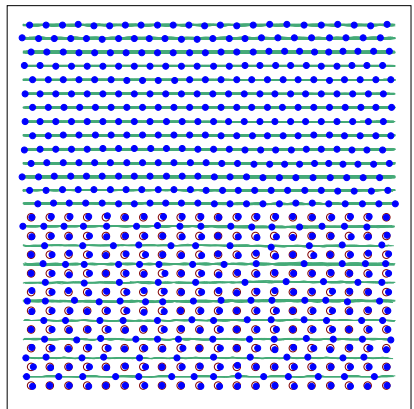

(b)
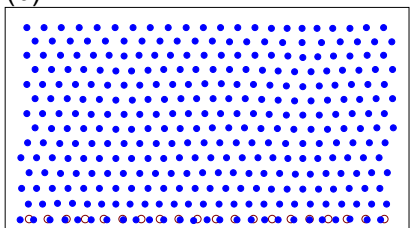

(n......................... . a.r........................ $\because \cdots \cdots \cdots \cdots \cdots \cdots$

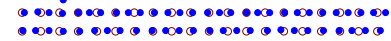
(d)

FIG. 4. The vortex positions (filled circles), pinning site positions (open circles), and vortex trajectories (lines) for the system in Fig. 1(b) with parallel driving at $B / B_{\phi}=1.67$. (a) At $F_{D}=0.1$, only the vortices in the unpinned region flow. (b) At $F_{D}=0.5$, the vortices in the unpinned region are flowing along with the interstitial vortices in the pinned region. (c) A plot of only the vortex positions at $F_{D}=1.2$, showing a coexistence between a moving hexagonal lattice in the upper, unpinned portion of the sample and a moving liquid or disordered state in the lower, pinned portion of the sample. (d) At $F_{D}=1.2$ and $B / B_{\phi}=1.33$, we find a moving lattice in the unpinned region and a moving smectic in the pinned region.

When $0.08<B / B_{\phi}<0.33$, we observe two stages of vortex flow. At lower drives, only the vortices in the unpinned region are moving, while at higher drives, above the step in $\left\langle V_{x}\right\rangle$, vortices in the pinned region depin and begin to move. Interstitial vortices first begin to penetrate the edge of the pinned region when $B / B_{\phi}>0.33$. The interstitial vortices are only indirectly pinned by interactions with the pinned vortices, and they depin near $F_{D}=0.15$. Their motion produces an intermediate flow state for $B / B_{\phi}>0.33$ in which plastic or disordered flow of vortices occurs in the pinned portion of the sample along with a more ordered flow of vortices in the unpinned portion of the sample. For $B / B_{\phi} \leq 0.08$, all of the vortices are pinned at low drives and there is a single depinning threshold at $F_{D} / F_{p}=1.0$. In Fig. 3(a) we illustrate the vortex trajectories for the system in Fig. 2 with $B / B_{\phi}=0.33$ at $F_{D}=0.5$, where only vortices in the pinned region are flowing. At $F_{D}=1.1$ in the same sample, Fig. 3(b) indicates that all the vortices are now flowing.

In Fig. 4(a) we show the motion in the sample from
Fig. 2 at $B / B_{\phi}=1.67$ and $F_{D}=0.1$. The vortices in the unpinned region are mobile and the vortices in the pinned region are immobile. The pinned region is filled with a combination of directly pinned vortices and interstitial vortices, whose motion is arrested only by interactions with the neighboring pinned vortices. In Fig. 4(b), the same sample at $F_{D}=0.5$ contains moving interstitial vortices in the pinned region, but the vortices at the pinning sites remain immobile. When $F_{D}>1.0$, all the vortices are moving; however, we find that there is a combination of a moving crystal state and a moving liquid state due to the inhomogeneous pinning, as shown in Fig. 4(c) at $F_{D}=1.2$. Here the vortices in the unpinned portion of the sample form a moving crystal while the vortices in the pinned region are disordered and constantly exchange neighbors to form a moving liquid state. A transverse pinning effect is responsible for the appearance of the moving liquid. Vortices in the pinned region preferentially move along the easy-flow one-dimensional (1D) channels separating neighboring rows of pinning sites; however, at this vortex density, the spacing between the vortices is not commensurate with the spacing between the easy-flow channels, so the system remains disordered. At higher drives than what we consider here, it is possible that the system could order into a moving lattice in the unpinned portion of the sample coexisting with a moving smectic state in the pinned portion of the sample. The ability of the vortices in the pinned region to dynamically order depends on the filling factor, and certain fillings permit ordered flows to occur in both the pinned and unpinned regions. An example of this appears in Fig. 4(d) for the same drive of $F_{D}=1.2$ as in Fig. 4(c) but at a field of $B / B_{\phi}=1.33$, where the vortices in the pinned region can form aligned $1 \mathrm{D}$ chains.

In Fig. 5 we plot a dynamic phase diagram as a function of $F_{D}$ versus $B / B_{\phi}$ for the system in Fig. 2 with parallel driving. Here Phase I corresponds to the state in which only the vortices in the pinned region are flowing, as shown in Fig. 3(a) and Fig. 4(a). The vertical dashed line indicates the point at which immobile interstitial vortices appear throughout the pinned region for $B / B_{\phi}>1.0$. In Phase $\mathrm{I}_{i n}$, both the vortices in the unpinned region and the interstitial vortices in the pinned region are flowing, while the pinned vortices remain immobile, as shown in Fig. 4(b). Additional commensurate-incommensurate transitions of the interstitial vortex structure can occur within phase $\mathrm{I}_{i n}$, producing small steps in the velocity-force curves (not shown). Phase In is an intermediate state in which different types of plastic flow occur. The vortices can be disordered or partially disordered within the pinned region or at the boundaries between the pinned and unpinned regions. The distinct types of plastic flow states that occur within Phase In are not discussed here but they have been outlined in previous work on samples that are filled with uniform periodic pinning arrays $34,36,37,39$. In Phase MS, all of the vortices are moving and a triangular lattice in the unpinned region coexists with a moving smec- 


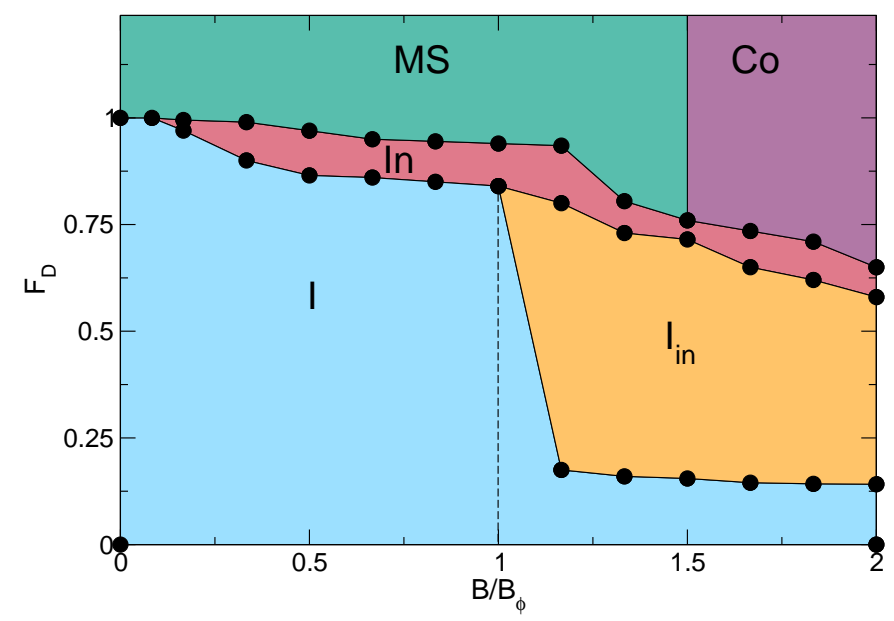

FIG. 5. A dynamic phase diagram as a function of $F_{D}$ vs $B / B_{\phi}$ for the system in Fig. 2 with parallel driving. In Phase I (blue), vortices are only flowing in the pin-free region. The dashed line indicates the point at which interstitial vortices appear throughout the pinned region in phase I when $B / B_{\phi}>1.0$. In Phase $\mathrm{I}_{i n}$ (orange), both the vortices in the unpinned region and the interstitial vortices in the pinned region are flowing, but the vortices in the pinning sites remain pinned, as illustrated in Fig. 4(b). Phase In (pink) consists of plastic or intermediate flow. In Phase MS (teal), all the vortices are moving and there is a combination of a moving triangular lattice in the unpinned region and a moving smectic in the pinned region, as shown in Fig. 4(d). Phase Co (purple) is a coexistence between a moving triangular lattice in the unpinned region and a moving liquid in the pinned

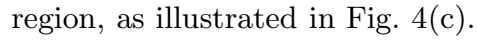

tic phase in the pinned region, as illustrated in Fig. 3(b) and Fig. 4(d). In Phase Co, all the vortices are moving and there is a coexistence between a moving triangular lattice in the unpinned region and a moving liquid in the pinned region.

\section{DRIVING IN THE PARALLEL DIRECTION AND JAMMED STATES}

We next consider driving in the positive $y$-direction, perpendicular to the pinning strip, which causes vortices in the unpinned region to move toward the the pinned region. In Fig. 6] we plot $\left\langle V_{y}\right\rangle$ vs $F_{D}$ for $B / B_{\phi}=0.083$, $0.167,0.33,0.5,0.67,0.83,1.167$, and 1.67 , where, unlike the case of parallel driving, we find that there is a finite depinning threshold for motion at all densities. Figure 6 indicates that the depinning threshold decreases with increasing $B / B_{\phi}$.

Below the depinning threshold, we draw a distinction between pinned and jammed phases. The pinned phase appears when $B / B_{\phi} \leq 0.5$, and is characterized by the direct pinning of vortices from the pin-free region by unoccupied pinning sites in the pinned region. At low drives, vortices in the unpinned region accumulate

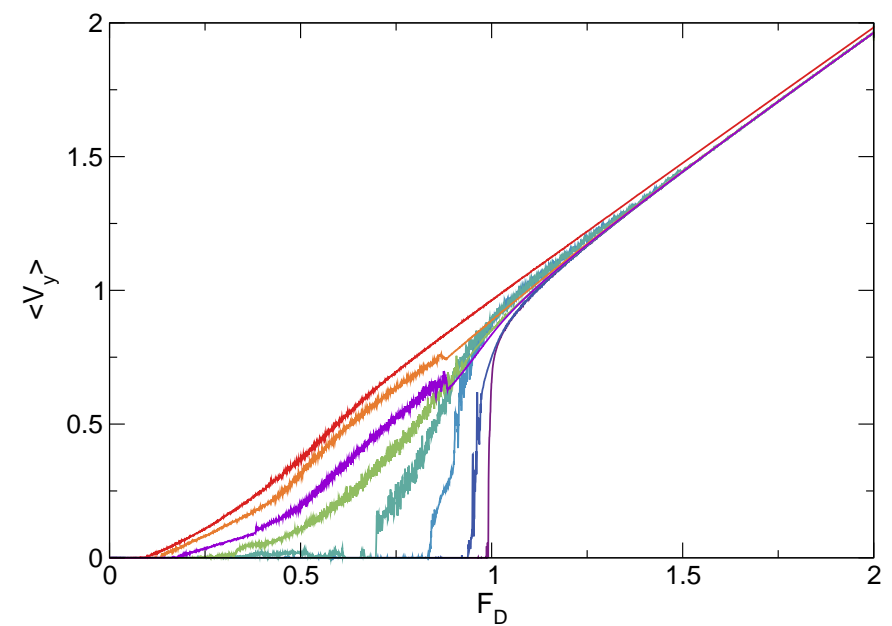

FIG. 6. $\left\langle V_{y}\right\rangle$ vs $F_{D}$ for the system in Fig. 1 for driving in the perpendicular direction at $B / B_{\phi}=0.083,0.167,0.33$, $0.5,0.67,0.83,1.167$, and 1.67, from bottom to top. For $B / B_{\phi}<0.5$, the system is pinned at low drives, while for $B / B_{\phi}>0.5$, the system is jammed at low drives.

along the edge of the pinned region and gradually force their way into the pinned region as the drive increases above $F_{D}=0.33$. As long as there are enough empty pinning sites to accommodate these vortices, $\left\langle V_{y}\right\rangle=0$. At $B / B_{\phi}=0.5$, we find a combination of jammed and pinned states in which a large portion of the vortices in the pin-free channel accumulate along the edge of the pinned region and force their way in, leading to a transient flow; however, most of the vortices can eventually find unoccupied pinning sites. These transients appear as a low level of flow near $F_{D}=0.5$ in the $B / B_{\phi}=0.5$ curve in Fig. 6] which is followed at higher drives by a collapse of $\left\langle V_{y}\right\rangle$ back to zero, indicating that all of the vortices have ceased to flow. At $B / B_{\phi}>0.55$, a clear jammed state emerges when all the pinning sites become occupied and the remaining vortices are immobilized only due to interactions with vortices in the pinned region. In systems that exhibit jamming, flow is blocked by interactions with other particles rather than being blocked by the direct trapping of particles by pinning sites 40,41 . As the vortex density increases, it becomes more difficult for the pinned vortices to hold back the vortices in the unpinned region, and the depinning threshold drops. Jamming phases in vortex systems have been discussed previously in the context of funnel geometries $\stackrel{42-45}{ }$ which contain constrictions that can block the vortex flow if the vortex density in the constriction becomes too large. Our results suggest that jamming can be observed clearly in a system with inhomogeneous pinning, and that jamming occurs when the number of vortices is larger than the number of pinning sites.

In Fig. 7 we illustrate the vortex and pinning site locations for a system with perpendicular driving at $B / B_{\phi}=1.167$. In Fig. 77(a), which shows the jammed state at $F_{D}=0.125$, vortices in the unpinned region pile 


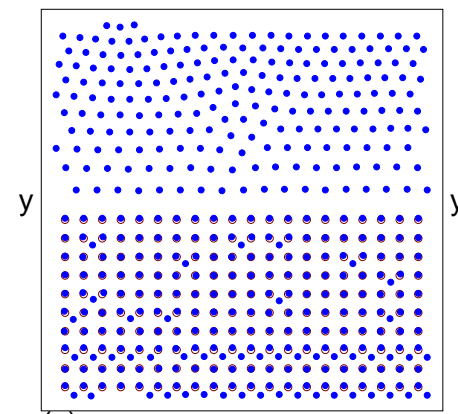

(a)

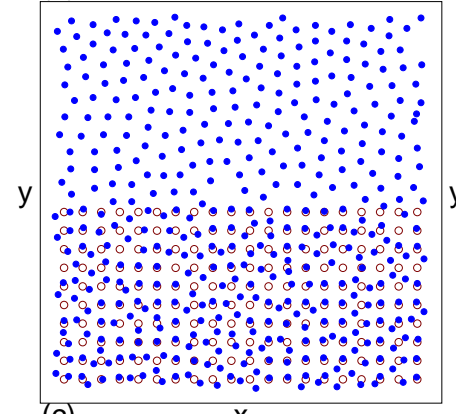

(c)

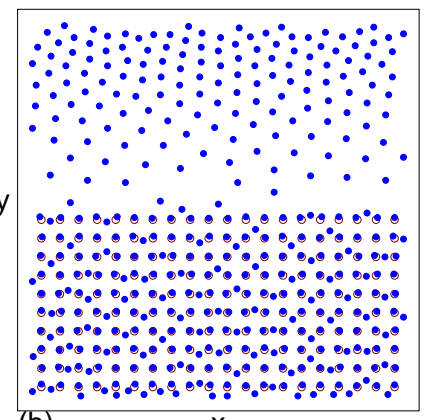

(b)

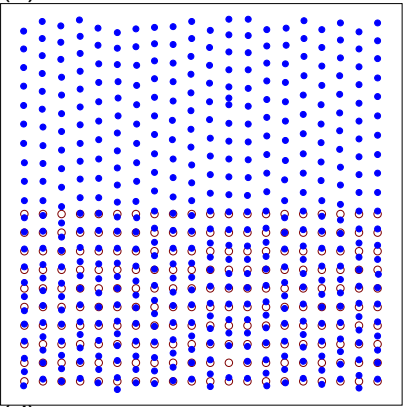

(d)

FIG. 7. The vortex positions (filled circles) and pinning site positions (open circles) for the system in Fig. 6 with perpendicular driving at $B / B_{\phi}=1.167$. (a) Jammed phase at $F_{D}=0.125$. (b) Flowing phase at $F_{D}=0.2$. (c) Disordered uniform flow phase at $F_{D}=0.75$. (d) Moving flow or moving smectic phase at $F_{D}=1.1$.

up behind the vortices in the pinned region, creating a vortex gradient in the unpinned region. At the onset of motion for $F_{D}=0.2$, Fig. 7 (b) indicates that there is still a gradient in the vortex density in the unpinned region, but that vortices can move through the pinned region by forming interstitials that slip between the pinning sites. At $F_{D}=0.75$ in Fig. 7( $(\mathrm{c})$, the flow is disordered and the gradient in the vortex density is lost. Finally, at $F_{D}=1.1$ in Fig. 7(d), the vortices have dynamically ordered into a moving smectic phase.

For $B / B_{\phi}>1.33$ under perpendicular driving, the jammed phase depins into a moving interstitial flow phase, where vortices can slip between the pinned vortices, and at higher drives the vortices at the pinning sites begin to depin, resulting in disordered flow. When $F_{D} / F_{p}>1.0$, the system can order into a moving smectic state. In Fig. 8(a) we illustrate the jammed phase for a sample with perpendicular driving at $B / B_{\phi}=1.67$, where we trace out the vortex trajectories over a fixed period of time. As the drive increases in the jammed phase, the vortices in the unpinned region gradually become more compressed, and in some cases they can undergo sudden avalanche-like rearrangements, but the motion always returns to zero in the long time limit. Figure 8 (b) shows the occurrence of interstitial flow at $F_{D}=0.2$, where the vortices follow $1 \mathrm{D}$ channels between the pinning sites. As the vortices reenter the pin-free region, the trajectories become more disordered and the vortices
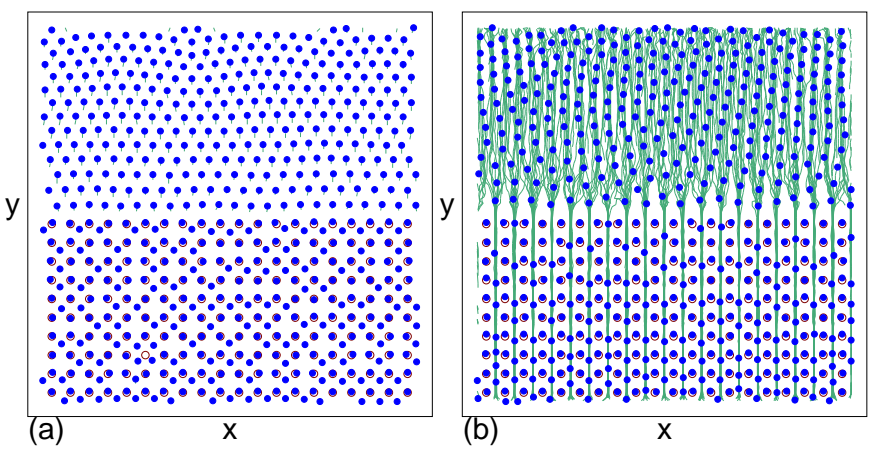

FIG. 8. The vortex positions (filled circles), pinning site positions (open circles), and vortex trajectories (lines) in a system with perpendicular driving at $B / B_{\phi}=1.67$. (a) The jammed phase at $F_{D}=0.1$. (b) The interstitial flow phase at $F_{D}=0.2$.

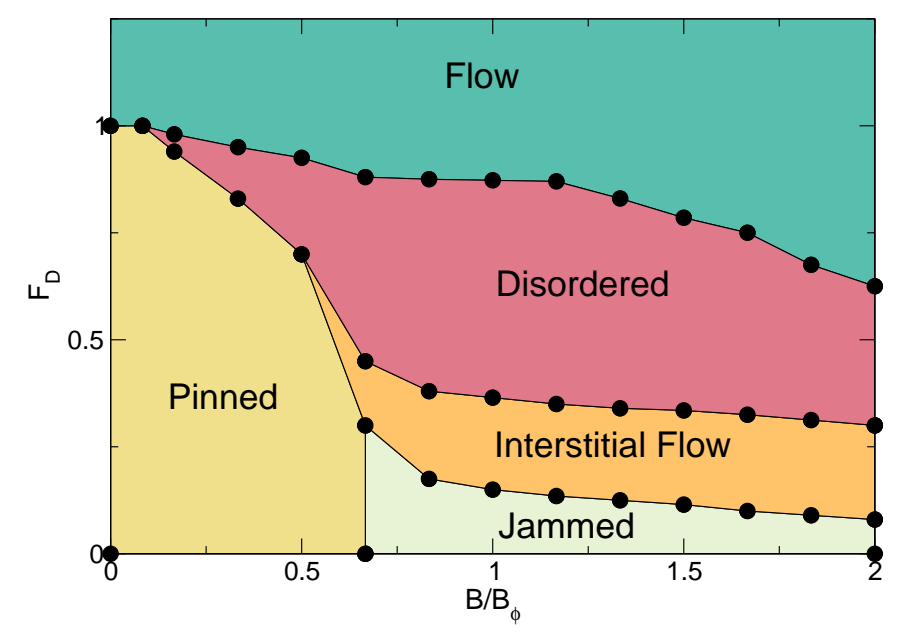

FIG. 9. A dynamic phase diagram as a function of $F_{D}$ vs $B / B_{\phi}$ for the system in Fig. 6 with perpendicular driving. Labels indicate the pinned phase (yellow), jammed phase (pale green), interstitial flow phase (orange), disordered flow phase (pink), and free flow or moving smectic phase (teal).

rearrange into a triangular lattice within the unpinned region.

In Fig. 9 we plot the dynamic phase diagram as a function of $F_{D}$ versus $B / B_{\phi}$ for the system in Fig. [6 with perpendicular driving. Labels indicate the pinned phase that appears for $B / B_{\phi}<0.67$, the jammed phase that occurs when $B / B_{\phi} \geq 0.67$, a disordered or intermediate phase composed of a mixture of pinned and flowing vortices, the interstitial flow phase which appears for drives just above the end of the jammed phase, and the free flow or moving smectic state at high drives.

Several additional effects could be studied for the jamming system. For example, the vortex gradient in the unpinned region has the same features as the conformal lattice that has been studied previously in vortex systems with density gradients 46,47 . Within the jammed phase, there could be avalanches with interesting properties that 


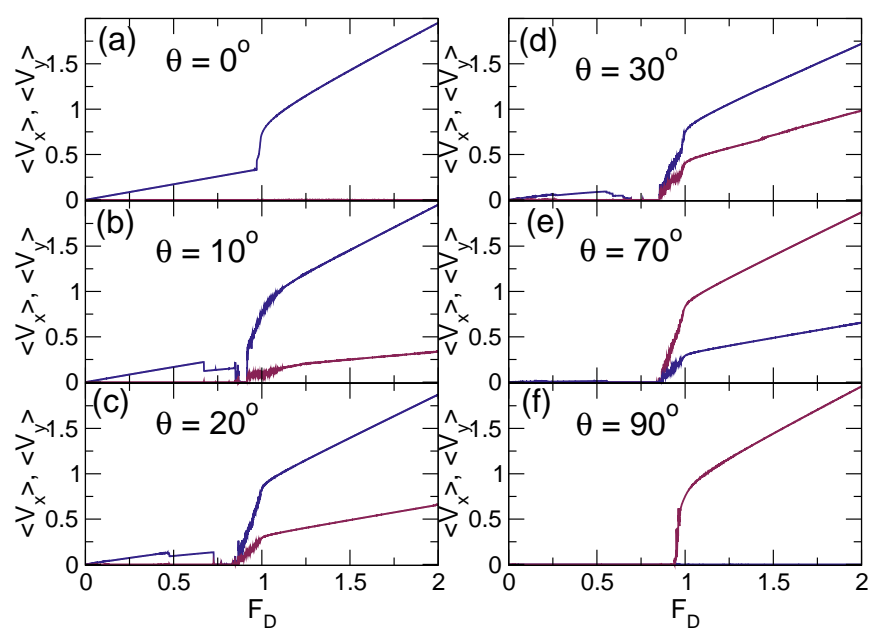

FIG. 10. $\left\langle V_{x}\right\rangle$ (blue) and $\left\langle V_{y}\right\rangle$ (red) vs $F_{D}$ for the system in Fig. 1 at $B / B_{\phi}=0.167$ under driving at varied angle $\theta$ with respect to the $x$ axis. Here $\theta=$ (a) $0^{\circ}$, (b) $10^{\circ}$, (c) $20^{\circ}$, (d) $30^{\circ}$, (e) $70^{\circ}$, and (f) $90^{\circ}$. A reentrant pinning regime appears at intermediate values of $\theta$.

occur when the system tries to simultaneously reduce the vortex lattice spacing while maintaining a triangular vortex lattice configuration.

\section{DRIVING AT AN ANGLE}

We next consider the case where the drive is applied at an angle $\theta$ with respect to the pinning strip. In Fig. 10 we focus on samples with a low vortex density of $B / B_{\phi}=$ 0.167. We plot $\left\langle V_{x}\right\rangle$ and $\left\langle V_{y}\right\rangle$ versus $F_{D}$ in Fig. [10(a) for $\theta=0^{\circ}$, where the two low density-dynamic phases I and MS described above in section III appear. At $\theta=10^{\circ}$, Fig. 10(b) indicates that the vortices are guided along the $x$ direction when they first begin to move, but as $F_{D}$ increases, there is a downward step in $\left\langle V_{x}\right\rangle$ followed by a drive-induced pinned region where both $\left\langle V_{x}\right\rangle$ and $\left\langle V_{y}\right\rangle$ are zero. Here, when $F_{D}$ is small, the vortices in the unpinned region undergo easy flow in the $x$-direction that is guided by the repulsion from the vortices trapped in the pinned region. As $F_{D}$ increases, the flowing vortices are eventually pushed into the pinned region, where they can each find an empty pinning site and become immobile, producing a drop in the vortex mobility. Since there are many more pinning sites than vortices, it is possible for all of the vortices to become trapped by pinning sites, producing the reentrant pinned phase. When the drive is high enough, the vortices at the pinning sites become depinned and both $\left\langle V_{x}\right\rangle$ and $\left\langle V_{y}\right\rangle$ begin to increase with increasing $F_{D}$. Similar behavior appears in Fig. 10 (c,d) for $\theta=20^{\circ}$ and $\theta=30^{\circ}$, with the width of the reentrant pinning regime increasing as $\theta$ increases. At $\theta=70^{\circ}$ in Fig. 10(e), the guided flow phase is almost completely lost, and at $\theta=90^{\circ}$ in Fig. 10(f), there is only a single pinned phase and the reentrance disappears.

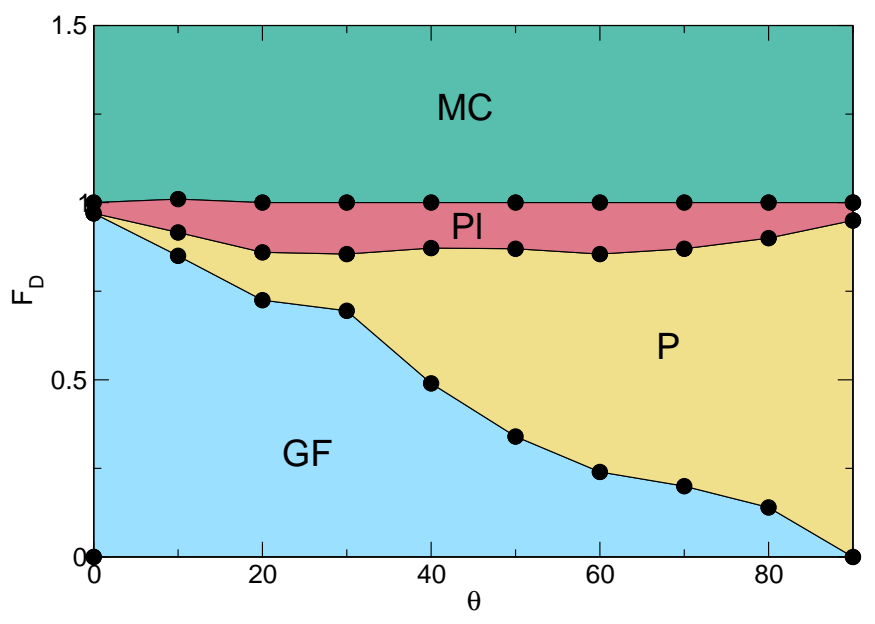

FIG. 11. A dynamic phase diagram as a function of $F_{D}$ vs $\theta$ for the system in Fig. 10 with $B / B_{\phi}=0.167$. Labels indicate the guided flow phase GF (blue), the pinned phase P (yellow), the intermediate or plastic flow phase $\mathrm{Pl}$ (pink), and the free flow phase MC (teal).

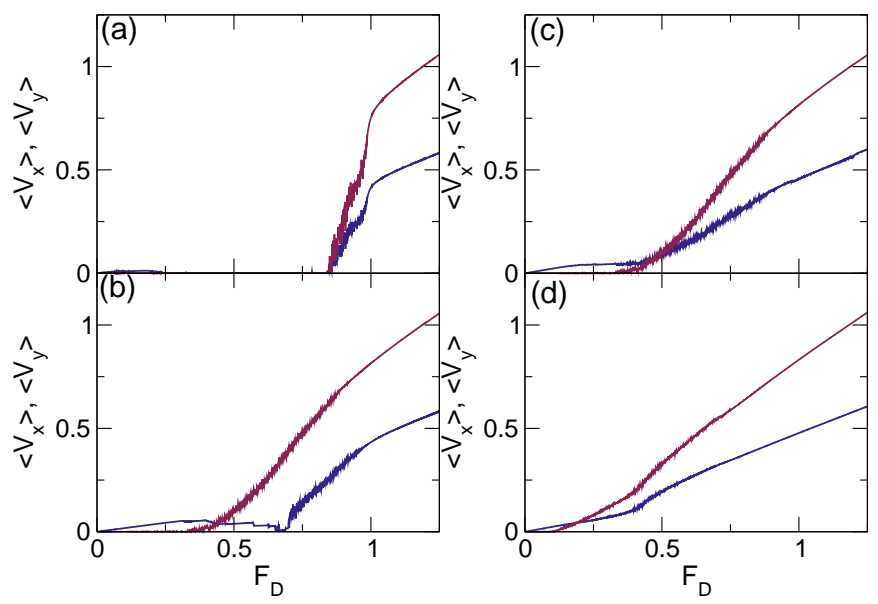

FIG. 12. $\left\langle V_{x}\right\rangle$ (blue) and $\left\langle V_{y}\right\rangle$ (red) vs $F_{D}$ for driving at $\theta=60^{\circ}$ with $B / B_{\phi}=$ (a) 0.167 , (b) 0.33 , (c) 0.67 , and (d) 1.67 .

In Fig. 11 we plot the dynamic phase diagram as a function of $F_{D}$ versus $\theta$ for the system in Fig. 10 with $B / B_{\phi}=0.167$. We find a guided flow phase, a pinned phase, a moving crystal or free flow phase at higher drives, and an intermediate or plastic phase between the pinned and free flow phases. The width of the reentrant pinned phase increases with increasing $\theta$.

The behavior of the reentrant pinning phase also depends on the filling factor. In Fig. [12(a) we plot $\left\langle V_{x}\right\rangle$ and $\left\langle V_{y}\right\rangle$ versus $F_{D}$ for the system in Fig. 10 at $B / B_{\phi}=0.167$ and $\theta=60^{\circ}$, which is in a pinned state at most low drives. When $B / B_{\phi}$ is increased to $B / B_{\phi}=0.33$, Fig. 12(b) indicates that an extended region emerges in which guided flow in the $x$-direction occurs. This is followed by a drive window over which $\left\langle V_{x}\right\rangle$ decreases with increasing $F_{D}$, 
indicating the appearance of negative differential conductivity, while $\left\langle V_{y}\right\rangle$ increases with increasing $F_{D}$. For $F_{D}>0.7$, both $\left\langle V_{x}\right\rangle$ and $\left\langle V_{y}\right\rangle$ increase with increasing $F_{D}$. For $F_{D}>1.0$, the velocity increase becomes linear with $F_{D}$ as the system enters the free flow limit. At $B / B_{\phi}=0.67$ in Fig. 12)(c), there is still a guided flow state at low drives, but the window of negative differential mobility is reduced in width. In Fig. 12(d) at $B / B_{\phi}=1.67$, guided flow appears for low $F_{D}$ where $\left\langle V_{y}\right\rangle=0$, and at higher drives, both velocity components increase monotonically with increasing $F_{D}$. We note that for certain driving angles, we also observe directional locking effects. We do not discuss these effects here, but similar features have been analyzed in previous woks 35,48 .

\section{SUMMARY}

We have numerically examined the dynamics of vortices in a system containing a square array of pinning sites where a strip of the pinning sites are removed in order to create an easy flow channel for vortex motion. When a driving force is applied parallel to the pinning strip, flow first occurs for the vortices in the unpinned region, followed by the depinning of vortices in the pinned region, leading to velocity-force curves with a two step feature. At high vortex densities we find additional phases including a multiple step depinning transition within the pinned region. For driving applied perpendicular to the pinning strip, we find that either a pinned or jammed state occurs at low drives depending on the vortex density. The jammed phase appears at higher magnetic fields when vortices in the unpinned region are blocked by the pinned vortices and accumulate at the edge of the pinned region, while at lower fields, a pinned state appears in which all of the vortices are eventually trapped directly by pinning sites. The crossover from the pinned to the jammed state appears as a decrease in the critical depinning force and an increase in the nonlinearity of the velocity-force curves. When the drive is applied at an angle to the pinning strip, we find a dynamically induced reentrant pinning, where vortices undergo guided flow at low drives due to the repulsion from the pinned vortices, but gradually enter the pinned region and become trapped as the driving force increases. If the number of vortices is smaller than the number of pinning sites, all of the vortices can become trapped, producing the reentrant pinned state. At higher vortex densities, the reentrant pinning effect is gradually replaced by a region of negative differential mobility and then by monotonic anisotropic flow. Our results could be tested using nanostuctured pinning arrays in which a portion of the pinning sites are omitted.

\section{ACKNOWLEDGMENTS}

This work was supported by the US Department of Energy through the Los Alamos National Laboratory. Los Alamos National Laboratory is operated by Triad National Security, LLC, for the National Nuclear Security Administration of the U. S. Department of Energy (Contract No. 892333218NCA000001).
1 S. Bhattacharya and M. J. Higgins, "Dynamics of a disordered flux line lattice," Phys. Rev. Lett. 70, 2617-2620 (1993).

2 C. J. Olson, C. Reichhardt, and F. Nori, "Nonequilibrium dynamic phase diagram for vortex lattices," Phys. Rev. Lett. 81, 3757-3760 (1998).

3 C. Reichhardt, C. J. Olson, N. Grønbech-Jensen, and F. Nori, "Moving Wigner glasses and smectics: Dynamics of disordered Wigner crystals," Phys. Rev. Lett. 86, 4354-4357 (2001).

4 N. Nagaosa and Y. Tokura, "Topological properties and dynamics of magnetic skyrmions," Nature Nanotechnol. 8, 899-911 (2013).

5 C. Reichhardt, D. Ray, and C. J. Olson Reichhardt, "Quantized transport for a skyrmion moving on a two-dimensional periodic substrate," Phys. Rev. B 91, 104426 (2015).

6 T. Bohlein, J. Mikhael, and C. Bechinger, "Observation of kinks and antikinks in colloidal monolayers driven across ordered surfaces," Nature Mater. 11, 126-130 (2012).

7 D. McDermott, J. Amelang, C. J. Olson Reichhardt, and C. Reichhardt, "Dynamic regimes for driven colloidal particles on a periodic substrate at commensurate and incommensurate fillings," Phys. Rev. E 88, 062301 (2013).
8 A. Vanossi, N. Manini, M. Urbakh, S. Zapperi, and E. Tosatti, "Colloquium: Modeling friction: From nanoscale to mesoscale," Rev. Mod. Phys. 85, 529-552 (2013).

9 O. Daldini, P. Martinoli, J. L. Olsen, and G. Berner, "Vortex-line pinning by thickness modulation of superconducting films," Phys. Rev. Lett. 32, 218-221 (1974).

10 R. Besseling, P. H. Kes, T. Drose, and V. M. Vinokur, "Depinning and dynamics of vortices confined in mesoscopic flow channels," New J. Phys. 7, 71 (2005)

11 K. Yu, T. W. Heitmann, C. Song, M. P. DeFeo, B. L. T. Plourde, M. B. S. Hesselberth, and P. H. Kes, "Asymmetric weak-pinning superconducting channels: Vortex ratchets," Phys. Rev. B 76, 220507 (2007).

${ }^{12}$ V. A. Shklovskij and O. V. Dobrovolskiy, "Influence of pointlike disorder on the guiding of vortices and the Hall effect in a washboard planar pinning potential," Phys. Rev. B 74, 104511 (2006).

13 V. A. Shklovskij and V. V. Sosedkin, "Guiding of vortices and ratchet effect in superconducting films with asymmetric pinning potential," Phys. Rev. B 80, 214526 (2009).

${ }^{14} \mathrm{~K} . \mathrm{Yu}, \mathrm{M}$. B. S. Hesselberth, P. H. Kes, and B. L. T. Plourde, "Vortex dynamics in superconducting channels with periodic constrictions," Phys. Rev. B 81, 184503 (2010). 
15 O. V. Dobrovolskiy, M. Huth, and V. A. Shklovskij, "Anisotropic magnetoresistive response in thin $\mathrm{Nb}$ films decorated by an array of Co stripes," Supercond. Sci. Technol. 23, 125014 (2010).

16 Q. Le Thien, D. McDermott, C. J. Olson Reichhardt, and C. Reichhardt, "Orientational ordering, buckling, and dynamic transitions for vortices interacting with a periodic quasi-one-dimensional substrate," Phys. Rev. B 93, 014504 (2016).

17 O. V. Dobrovolskiy, "Abrikosov fluxonics in washboard nanolandscapes," Physica C 533, 80-90 (2017).

18 O. V. Dobrovolskiy, M. Huth, V. A. Shklovskij, and R. V. Vovk, "Mobile fluxons as coherent probes of periodic pinning in superconductors," Sci. Rep. 7, 13740 (2017)

19 O. V. Dobrovolskiy, V. A. Shklovskij, M. Hanefeld, M. Zörb, L. Köhs, and M. Huth, "Pinning effects on flux flow instability in epitaxial $\mathrm{Nb}$ thin films," Supercond. Sci. Technol. 30, 085002 (2017).

20 O.V. Dobrovolskiy, V.M. Bevz, E. Begun, R. Sachser, R.V. Vovk, and M. Huth, "Fast dynamics of guided magnetic flux quanta," Phys. Rev. Applied 11, 054064 (2019).

21 M. C. Marchetti and D. R. Nelson, "Patterned geometries and hydrodynamics at the vortex Bose glass transition," Phys. Rev. B 59, 13624-13627 (1999)

22 M. Basset, G. Jakob, G. Wirth, and H. Adrian, "Patterned irradiation of $\mathrm{YBa}_{2} \mathrm{Cu}_{3} \mathrm{O}_{7-x}$ thin films," Phys. Rev. B 64, 024525 (2001).

23 W. K. Kwok, R. J. Olsson, G. Karapetrov, U. Welp, V. Vlasko-Vlasov, K. Kadowaki, and G. W. Crabtree, "Modification of vortex behavior through heavy ion lithography," Physica C 382, 137-141 (2002)

24 A. Crassous, R. Bernard, S. Fusil, K. Bouzehouane, D. Le Bourdais, S. Enouz-Vedrenne, J. Briatico, M. Bibes, A. Barthélémy, and J. E. Villegas, "Nanoscale electrostatic manipulation of magnetic flux quanta in ferroelectric/superconductor $\mathrm{BiFeO}_{3} / \mathrm{YBa}_{2} \mathrm{Cu}_{3} \mathrm{O}_{7-\delta}$ heterostructures," Phys. Rev. Lett. 107, 247002 (2011)

25 O. V. Dobrovolskiy, E. Begun, M. Huth, and V. A. Shklovskij, "Electrical transport and pinning properties of $\mathrm{Nb}$ thin films patterned with focused ion beam-milled washboard nanostructures," New J. Phys. 14, 113027 (2012).

26 J. Trastoy, V. Rouco, C. Ulysse, R. Bernard, G. Faini, J. Lesueur, J. Briatico, and J. E. Villegas, "Nanostructuring of high- $T_{C}$ superconductors via masked ion irradiation for efficient ordered vortex pinning," Physica C 506, 195-200 (2014)

27 R. Seshadri and R. M. Westervelt, "Forced shear flow of magnetic bubble arrays," Phys. Rev. Lett. 70, 234-237 (1993).

28 C. Reichhardt and C. J. O. Reichhardt, "Chiral edge currents for ac-driven skyrmions in confined pinning geometries," Phys. Rev. B 100, 174414 (2019)

29 M. Baert, V. V. Metlushko, R. Jonckheere, V. V. Moshchalkov, and Y. Bruynseraede, "Composite flux-line lattices stabilized in superconducting films by a regular array of artificial defects," Phys. Rev. Lett. 74, 3269-3272 (1995).

30 J. I. Martín, M. Vélez, J. Nogués, and I. K. Schuller, "Flux pinning in a superconductor by an array of submicrometer magnetic dots," Phys. Rev. Lett. 79, 1929-1932 (1997)

31 C. Reichhardt, C. J. Olson, and F. Nori, "Commensurate and incommensurate vortex states in superconductors with periodic pinning arrays," Phys. Rev. B 57, 7937-7943 (1998).

32 G. R. Berdiyorov, M. V. Milošević, and F. M. Peeters, "Novel commensurability effects in superconducting films with antidot arrays," Phys. Rev. Lett. 96, 207001 (2006).

33 I. Swiecicki, C. Ulysse, T. Wolf, R. Bernard, N. Bergeal, J. Briatico, G. Faini, J. Lesueur, and J. E. Villegas, "Strong field-matching effects in superconducting $\mathrm{YBa}_{2} \mathrm{Cu}_{3} \mathrm{O}_{7-\delta}$ films with vortex energy landscapes engineered via masked ion irradiation," Phys. Rev. B 85, 224502 (2012)

34 C. Reichhardt, C. J. Olson, and F. Nori, "Dynamic phases of vortices in superconductors with periodic pinning," Phys. Rev. Lett. 78, 2648-2651 (1997)

35 C. Reichhardt and F. Nori, "Phase locking, devil's staircases, Farey trees, and Arnold tongues in driven vortex lattices with periodic pinning," Phys. Rev. Lett. 82, 414-417 (1999)

36 J. Gutierrez, A. V. Silhanek, J. Van de Vondel, W. Gillijns, and V. V. Moshchalkov, "Transition from turbulent to nearly laminar vortex flow in superconductors with periodic pinning," Phys. Rev. B 80, 140514 (2009).

37 S. Avci, Z. L. Xiao, J. Hua, A. Imre, R. Divan, J. Pearson, U. Welp, W. K. Kwok, and G. W. Crabtree, "Matching effect and dynamic phases of vortex matter in $\mathrm{Bi}_{2} \mathrm{Sr}_{2} \mathrm{CaCu}_{2} \mathrm{O}_{8}$ nanoribbon with a periodic array of holes," Appl. Phys. Lett. 97, 042511 (2010).

38 R. M. da Silva and C. C. de Souza Silva, "Vortex density waves and negative absolute resistance in patterned superconductors," Phys. Rev. B 83, 184514 (2011).

39 C. Reichhardt and C. J. Olson Reichhardt, "Depinning and nonequilibrium dynamic phases of particle assemblies driven over random and ordered substrates: a review," Rep. Prog. Phys. 80, 026501 (2017)

40 A. J. Liu and S. R. Nagel, "The jamming transition and the marginally jammed solid," in Annual Review of Condensed Matter Physics, Vol. 1 edited by J.S. Langer (2010) pp. 347-369.

41 C. Reichhardt and C. J. Olson Reichhardt, "Aspects of jamming in two-dimensional athermal frictionless systems," Soft Matter 10, 2932-2944 (2014)

42 C. J. Olson Reichhardt and C. Reichhardt, "Commensurability, jamming, and dynamics for vortices in funnel geometries," Phys. Rev. B 81, 224516 (2010).

43 G. Karapetrov, V. Yefremenko, G. Mihajlović, J. E. Pearson, M. Iavarone, V. Novosad, and S. D. Bader, "Evidence of vortex jamming in Abrikosov vortex flux flow regime," Phys. Rev. B 86, 054524 (2012)

44 V. Vlasko-Vlasov, T. Benseman, U. Welp, and W. K. Kwok, "Jamming of superconducting vortices in a funnel structure," Supercond. Sci. Technol. 26, 075023 (2013)

45 C. J. O. Reichhardt and C. Reichhardt, "Clogging and transport of driven particles in asymmetric funnel arrays," J. Phys.: Condens. Matt. 30, 244005 (2018)

46 D. Ray, C. J. Olson Reichhardt, B. Jankó, and C. Reichhardt, "Strongly enhanced pinning of magnetic vortices in type-II superconductors by conformal crystal arrays," Phys. Rev. Lett. 110, 267001 (2013)

47 R. M. Menezes and C. C. de Souza Silva, "Conformal vortex crystals," Sci. Rep. 7, 12766 (2017)

48 C. Reichhardt and C. J. Olson Reichhardt, "Moving vortex phases, dynamical symmetry breaking, and jamming for vortices in honeycomb pinning arrays," Phys. Rev. B 78, 224511 (2008) 